\begin{tabular}{lllllllllllllllllllllllllllllllll}
\hline$R$ & $E$ & $V$ & I & S & T & A & D & E & E & S & T & U & D & I & O & S & I & N & T & E & R & N & A & C & I & O & N & A & L & E & S
\end{tabular}

\title{
Visión estratégica de la Armada de Chile y de su relación con Perú
}

Miguel A. Vergara

Conferencia en la Academia de Guerra Naval del Perú, 25 de junio de 2002.

\section{Palabras iniciales}

Constituye un alto honor, para quien les habla, ocupar la tribuna de esta Escuela Superior de Guerra Naval y dirigirme a este selecto auditorio, encabezado por el Comandante General de la Marina de Guerra del Perú, Almirante Don Ricardo Arboccó Liceti, con la presencia de parte importante de su Alto Mando. Ésta es una oportunidad que me parece nunca antes se había producido, lo que nos ofrece un indicio respecto del creciente acercamiento entre nuestras Armadas.

Soy consciente de que aún queda un largo camino por recorrer para alcanzar un nivel de cooperación como el que los tiempos exigen. Vamos por el camino correcto pero, quizás, deberíamos apurar el ritmo, puesto que Perú y Chile se necesitan mutuamente para ocupar el espacio que nuestras Patrias merecen en el mundo que se está construyendo y tengo la íntima convicción de que la cooperación es la única manera de lograrlo. 
Visión estratégica

de la Armada

de Chile
En efecto, nos encontramos en un momento histórico en que el mundo avanza rápido; en que la nueva situación mundial cada día se delinea y consolida; en que las necesidades de nuestras sociedades son más grandes y urgentes; y en que las oportunidades aparecen, pasan y se desvanecen a alta velocidad.

\section{Nuestro PASAdo}

Para enfrentar juntos este desafío que nos impone la globalización, propongo empezar por despejar algunas percepciones distintas de nuestro pasado común. Pasado que es importante, ya que contiene la memoria histórica y de algún modo contribuye a determinar lo que somos y cómo somos.

Nuestra historia común es variada y tiene momentos de cooperación y convergencia de propósitos, y momentos de conflicto y desencuentro. A mi memoria viene el Imperio Inca, cuya cultura superior se puede reconocer en caminos y pucarás que aún existen hasta en la parte centro norte de Chile; esas obras dan testimonio de la intensidad con que la presencia de vuestros antepasados se manifestó en nuestro país. Posteriormente, y desde el inicio mismo del proceso de conformación de nuestra nacionalidad, la presencia colonial del Virreinato y específicamente de las ciudades de Lima y Callao, fueron los referentes principales de la vida económica, social y cultural de nuestra Capitanía General.

Más adelante, en la lucha de nuestros pueblos por la Independencia de España, se generó una natural y espontánea cooperación fraternal que nos permitió compartir próceres militares y líderes políticos. Lamentablemente, a poco andar tuvimos nuestro primer desencuentro, en la Guerra entre Chile y la Confederación Perú-Boliviana, la cual afortunadamente no dejó huellas profundas en el alma de nuestras Naciones. Prueba de ello fue la comunión de ideales que se produjo a raíz de la Guerra contra España, en 1865 , que vio combatir y recibir juntos su bautismo de fuego a quienes posteriormente serían nuestros máximos héroes navales: Miguel Grau y Arturo Prat.

Después, a fines del siglo XIX, ocurrió el más profundo desencuentro de nuestros países en lo que conocemos como la Guerra del Pacífico. Es evidente que tenemos diferentes percep- 
ciones de esa guerra y sus efectos. Lamentablemente, los hechos del pasado no pueden ser cambiados, son hechos que sucedieron y nada puede hacerse para reemplazarlos. No obstante, y sin perder un ápice de objetividad, podemos hacer un esfuerzo por interpretar aquellos mismos hechos desde otra perspectiva; y eso sí que es materia de nuestra elección.

Sugiero comenzar reconociendo y aceptando la sinceridad y honestidad de las razones esgrimidas por ambos países. Puede que hoy los argumentos y motivaciones de los contendores de aquella época no nos resulten convincentes, pero no cabe duda de que, en la oportunidad, ambos pueblos los creyeron válidos y de tal importancia como para aceptar incluso el sacrificio supremo.

También es digno de destacar que en aquel conflicto, ambos países lucharon como lo hacen los mejores: hasta alcanzar la victoria o morir en el intento. Felicitémonos por haber sido dignos adversarios, por haber luchado con coraje y entereza por una causa que creímos justa. Ello nos permite respetarnos mutuamente y mirarnos a la cara sin reservas, más allá de las mutuas victorias y derrotas de aquel largo conflicto.

Por nuestra parte, la Armada de Chile estima que se prestigia y enaltece a sí misma cuando honra a las personas, las instituciones y los Estados que en algún momento fueron sus rivales. Por eso hoy, en esta Escuela Superior de Guerra Naval, quiero recordar al Gran Almirante Don Miguel Grau y Seminario, marino ejemplar, estratega genial y un caballero a carta cabal; en una palabra un señor del mar, digno ejemplo para generaciones futuras de marinos, no sólo del Perú. Verdaderamente sentimos que el Almirante Grau y la Marina de Guerra del Perú son actores brillantes de nuestra propia historia, con quienes compartimos nuestras glorias y también nuestros dolores y frustraciones.

\section{NUESTRO FUtURO}

Afortunadamente, con posterioridad a la Guerra del Pacífico hemos sabido vivir en paz y resolver nuestras controversias mediante el diálogo; y confiamos en que esta actitud continuará en el futuro. No obstante, más allá de haber sido capaces de conservar el valioso bien de la paz, es un hecho que los procesos de desarro- 
Visión estratégica

de la Armada

de Chile llo y consolidación de las identidades nacionales de Perú y Chile, han marchado por 123 años más bien por caminos paralelos y, a veces, divergentes. El desafío es que en el futuro próximo, nuestros caminos converjan hacia expectativas e intereses comunes. Ésta es una tarea ardua que requiere de voluntad para construir una relación de mayor confianza, lo que implica hacer un esfuerzo por querernos más.

En tal sentido, teniendo en cuenta que no se puede querer lo que no se conoce, creo que este cambio debe comenzar por una exploración y conocimiento mutuo que permita despejar prejuicios anclados en la historia, de modo de enfrentar juntos las realidades del presente y las expectativas del futuro. Ésta es la razón por la que creo que el intercambio de oficiales alumnos entre nuestras Academias de Guerra Naval es tan importante y que debemos cuidarlo e intensificarlo. Incluso, deberíamos ampliar estas actividades de intercambio hacia el personal subalterno y a los intelectuales navales que pertenecen al mundo de los marinos en retiro. Todos debemos poner nuestra buena voluntad y esfuerzo.

Nuestra participación activa es fundamental si pretendemos tener éxito en este proceso que tiene mucho de cambio cultural.

Queremos que ustedes nos conozcan, y es por eso que en esta solemne oportunidad deseo explicarles, con la mayor transparencia, cuál es el camino que hemos emprendido, y que desearíamos recorrer conjuntamente con Perú. Para ello primero me referiré brevemente al proyecto nacional de Chile y a su estrategia. En segunda instancia, situaré a la Armada en el contexto anterior, con sus objetivos y estrategia institucional. Concluiré esbozando cómo visualizamos la relación entre nuestros dos países y entre nuestras Armadas.

\section{Proyecto y estrategia Nacional en Chile}

Nuestro Proyecto Nacional, que probablemente en sus enunciados es similar al de ustedes, aspira a proporcionar a todos los chilenos educación de buena calidad, que les permita desarrollarse como personas, sin otros límites que sus propias capacidades; acceder a la modernidad y a la globalización en condiciones de igualdad con los miembros de sociedades más desarrolladas; ac- 
ceder a servicios de salud eficaces y al alcance de todos; acceder a los bienes de consumo que les permitan una calidad de vida digna de un habitante del mundo en el siglo XXI, en un contexto social más solidario, equitativo y justo.

Para llevar a cabo su proyecto-país, Chile en su Política interna optó por consolidar una democracia representativa y, en lo económico, por una economía social de mercado abierta al mundo. En política exterior sostenemos la igualdad de los Estados, el respeto a los tratados, la solución pacífica de las controversias, y la participación activa en la mantención y promoción de la paz. Consecuentemente, basamos nuestra política de Defensa en la disuasión y la cooperación.

Nuestra estrategia para materializar este proyecto está centrada en alcanzar el bienestar y seguridad de las personas. En tal sentido, nuestras Políticas Exterior y de Defensa las hemos puesto al servicio del desarrollo socioeconómico del país, en un contexto de apertura al mundo en lo económico, en lo social y en lo cultural, con todos los riesgos y beneficios que esa decisión conlleva.

En este contexto, el presidente de la República, Don Ricardo Lagos Escobar, en su mensaje anual a la Nación, el 21 de mayo recién pasado, expresó: "Mi deber como presidente es trabajar para que Chile entre al mundo global aprovechando al máximo las oportunidades que se nos ofrecen, disminuir los riesgos y cuidar que los beneficios de esa globalización se distribuyan equitativamente entre todas las familias. Chile es un país pequeño y lejano, siempre lo ha sido. Pero ni la lejanía nos debe empujar al aislamiento, ni lo pequeño de nuestro mercado hacia el proteccionismo. Mi gobierno no está disponible para políticas aislacionistas ni proteccionistas, no importa las presiones que debamos enfrentar".

Desde esta perspectiva, Chile para prosperar necesita que el entorno conformado por la región, el hemisferio y el mundo, sea también próspero y seguro. Es decir, nuestra estrategia nos impone una actitud de participación internacional, y de cooperación con otros Estados con los que compartimos intereses.

Por eso planteamos que la estabilidad, la prosperidad y el progreso de nuestros vecinos son importantes para Chile, ya que en esta aventura del siglo XXI que recién comienza, querámoslo o 
Visión estratégica

de la Armada

de Chile no, estamos inevitablemente asociados. En el mundo globalizado en que vivimos, los éxitos de nuestros vecinos son los éxitos de Chile y viceversa, pues, contribuyen a conformar una región mejor posicionada, digna de un mayor respeto internacional, y por consiguiente, capaz de generar un beneficio directo para todos los países de la región.

Visualizamos la reciente asociación de Chile con la Unión Europea como una avanzada de futuros acuerdos de otros países de la región con ese organismo, que permitan ir conformando una red de interrelaciones económicas y políticas que otorguen a la región una cada vez mayor estabilidad, mayor libertad de acción y mayor voz en los asuntos internacionales. Lo mismo vale para el Tratado de Libre Comercio con los Estados Unidos, proceso actualmente en un avanzado estado de negociación.

Como ustedes pueden apreciar, desde la perspectiva de nuestra Estrategia Nacional, los verbos a conjugar son "integración y cooperación". Queremos, a nivel regional, avanzar juntos hacia un nivel más alto de relaciones entre nuestros países; y esto pasa por el éxito que alcancemos en la búsqueda de mecanismos de solución pacífica de los eventuales conflictos de intereses que pudieran existir o sobrevenir en la región y, fundamentalmente, en que seamos capaces de conservar y consolidar estas soluciones en el tiempo.

Debemos considerar que los factores que determinan la riqueza de las naciones en el mundo actual están referidos más a la solidez de sus instituciones, a la educación y salud de sus habitantes y a la calidad de la gestión de sus gobiernos, que a la posesión de territorios, de riquezas naturales o de materias primas.

\section{La Armada de Chile en el CONTEXTo del Proyecto Nacional}

La Estrategia Nacional de desarrollo que he reseñado se conformó paulatinamente a partir de los objetivos del Proyecto Nacional que ya señalé. Estos procesos fueron avanzando sistemáticamente, pero se aceleraron a partir del término de la Guerra Fría y comienzo de la globalización.

La Armada de Chile se hizo cargo de los cambios ocurridos, y a partir de las políticas de gobierno y de las características más 
permanentes del Proyecto Nacional, hizo una propuesta de solución, que ha sido recogida, en sus elementos más relevantes, por la comunidad académica y por los actores políticos.

Con relación a esto, creo conveniente destacar algunos aspectos que podrían ser de interés para este ilustrado auditorio naval. El primero de ellos es el hecho de que en las nuevas circunstancias, determinadas por el contexto internacional actual, las Armadas en general y la Armada de Chile en particular, tienen una misión que no se restringe sólo a la defensa del territorio nacional. Por el contrario, viviendo en un mundo globalizado, y con una economía abierta como la nuestra, en que el $87 \%$ del comercio exterior se efectúa por mar, y casi el $60 \%$ de nuestro PIB. está relacionado con tal comercio, la Armada pasa a ser un elemento importante en la presencia y acción internacional de Chile.

Sin desconocer que nuestra misión prioritaria sigue siendo la defensa del territorio y de la soberanía Nacional, nos hemos abierto a incluir la defensa de intereses, dondequiera que ellos se expresen. Sin embargo, para proteger los intereses comerciales de ultramar, dado nuestros limitados medios, resulta imperativo actuar en conjunto con otros países. De allí nace el interés por desarrollar en mayor grado que antes nuestra capacidad de interoperación con otras Marinas y crear capacidades estratégicas acordes al tipo de participación internacional a que aspiramos.

De lo anterior deriva nuestro esfuerzo por participar en ejercicios multinacionales como RIMPAC, TEAMWORK NoRTH, TEAMWORK South y MARCot, que realizamos alternativamente cada dos años. Asimismo, en el ámbito regional participamos en UnITAs Pacífico, ejercicios Reskatamuy con Perú, VieKaren e InTEgración con Argentina, y Bogatun con Brasil. A ellos se agregan los ejercicios de oportunidad PASSEX que efectuamos con los buques de marinas amigas que nos visitan.

En concreto, estamos en un proceso de ampliar nuestro foco de atención, desde la defensa territorial hacia la participación marítima internacional; desde nuestra preocupación por una eventual confrontación con los Estados vecinos, hacia la cooperación con todos los países con quienes compartimos valores e intereses, sean estos vecinos o ubicados en ultramar. Esta mirada oceánica, que seguramente compartimos, nos abre, como Marinas, un mundo nuevo, apasionante y de real utilidad para los intereses nacio- 
Visión estratégica de la Armada de Chile nales de nuestros respectivos países, así como una oportunidad de cooperación de dimensiones insospechadas.

A este respecto, quisiera citar las palabras de la Sra. ministra de Defensa Nacional, Doctora Michelle Bachelet Jeria, en su discurso inaugural del Mes del Mar, en mayo pasado, en el contexto de un seminario organizado por la Marina, titulado "La Armada en la presencia internacional de Chile; un aporte real". Allí la ministra expresó: "Lo que finalmente ha ocurrido es un proceso en el cual nuestros conceptos y políticas de Defensa se encuentran transitando desde una aproximación histórica esencialmente territorial, hacia otra que expresa una ampliación de nuestro interés Nacional hacia dimensiones regionales y globales".

En definitiva, creemos que estamos en un nuevo escenario, en que la transparencia, las medidas de confianza mutua y también la disuasión, han contribuido a que tengamos la percepción de que la probabilidad de ocurrencia de conflictos vecinales sea, para Chile, más baja que en décadas anteriores. Y queremos que esa probabilidad tienda a cero; de allí nuestro interés por reforzar aún más los lazos de cooperación con nuestros vecinos. Esto explica en gran parte mi presencia ante este distinguido auditorio, en la certeza que en lo esencial compartimos esta visión de promover una mayor cooperación entre nuestros países, no sólo en el ámbito de la Defensa.

\section{ESTRATEGIA DE LOS TRES VECTORES}

En el contexto que hemos descrito, la propuesta de la Armada de Chile fue el planteamiento de una estrategia naval y marítima que denominamos "Estrategia de los Tres Vectores", que apunta al establecimiento del Control del Mar en tres condiciones o situaciones diferentes.

El primer vector lo constituye la defensa de nuestro territorio nacional, de sus ciudadanos, de sus bienes y de sus derechos. En este ámbito, la Institución es parte de un sistema militar, que en conjunto con el Ejército y con la Fuerza Aérea, actúa para disuadir o rechazar a un eventual agresor. Este vector es de naturaleza conjunta y abarca todo el territorio nacional. La tarea de la Arma- 
da es controlar el mar en un determinado espacio, durante el tiempo que lo requieran las operaciones en desarrollo.

El segundo vector lo constituye la defensa y control del amplio espacio jurisdiccional marítimo de Chile. Nuestra tarea es proteger la vida humana en el mar y los recursos vivos renovables que allí existen; salvaguardar el medio ambiente marino y hacer cumplir las leyes de la república. En síntesis, la Institución se empeña en contribuir a proporcionar mares limpios y seguros que faciliten el desarrollo económico del país, promoviendo el uso y explotación racional del enorme potencial económico, turístico y deportivo-recreativo de nuestro mar. Complementando lo anterior, realizamos tareas de seguridad policial y prevención de riesgos en los recintos portuarios y playas, apoyamos la seguridad de la navegación mediante la producción de cartografía náutica, la mantención de la señalización marítima y la administración del servicio de practicaje y pilotaje. Además, participamos en la realización o el apoyo de estudios científicos en los ámbitos de la oceanografía y meteorología, entre otros, cuyo aporte facilita las actividades industriales y comerciales en el mar.

El tercer vector lo constituye la participación en la promoción y protección de un interés nacional prioritario y fundamental, como es la conservación de la paz, la seguridad, y la estabilidad del orden internacional. Es decir, la promoción y defensa de las condiciones que permitan el libre tránsito de bienes y de personas, a través del mar, lo que estimamos es consustancial a nuestro desarrollo y progreso. Cabe destacar que en este ámbito, propio de la seguridad internacional, planteamos la promoción de intereses, además de su defensa. Esto nos permite abrirnos a una seguridad no sólo focalizada en las amenazas, sino también en la identificación de oportunidades que, a su vez, pueden generar nuevos intereses por proteger.

En este tercer vector, las doctrinas, procedimientos y capacidades, están orientadas a operar con unidades y fuerzas navales de otros países, formando parte de una coalición que comparta nuestros intereses. Previa una decisión política, debemos estar en condiciones de concurrir a cualquier lugar del mundo, y enfrentar amenazas que serían imposibles de controlar sólo con los medios propios. 
Visión estratégica

de la Armada

de Chile
7. Renovación del Poder Naval

De la estrategia antes enunciada se desprenden las necesidades de personal y material de la Armada de Chile. Las tareas por desarrollar, en el ámbito nacional e internacional, son múltiples y quisiéramos tener más medios para satisfacerlas. No obstante, somos conscientes de los recursos que disponemos y de las prioridades sociales de nuestro gobierno.

Por eso, nuestra meta se limita a renovar las unidades de nuestra Escuadra que han cumplido 30 o más años de servicios y están entrando en obsolescencia técnica y logística. Ni siquiera estamos renovando todas las unidades que hemos dado de baja. Objetivamente nos hemos reducido, puesto que hace sólo seis años contábamos con 10 unidades de combate, entre destructores y fragatas, y hoy tenemos sólo seis. Esperamos en el mediano plazo llegar a ocho.

En cuanto a la Fuerza de Submarinos, las nuevas unidades que estamos construyendo con el consorcio Franco-Español DCN - IZAR, reemplazarán a los submarinos Haytt y O'Brien, recientemente dados de baja del servicio.

Ésta es nuestra realidad; y les digo responsablemente que la Armada de Chile no está en carrera armamentista alguna.

\section{8. ÁREAS DE COOPERACIÓN EN EL ÁMBITO MARÍTIMO Y NAVAL}

Lejos de una carrera armamentista, estamos en un proceso de apertura internacional y de cooperación. En este esquema, los quisiera invitar a reflexionar respecto a las eventuales actividades que Perú y Chile podrían explorar o intensificar en forma mancomunada en el entorno marítimo y naval.

En el pasado reciente, actuando conjuntamente en el campo internacional, hemos alcanzado logros espectaculares. Tal vez el mayor y que más satisfacciones puede habernos dado es el obtenido por Perú, Chile y Ecuador en agosto de 1952, cuando firmamos la Declaración de Santiago, que vino a formalizar las declaraciones de los respectivos Presidentes en el año 1947, fijando nuestros derechos sobre los espacios marítimos hasta las 200 mi- 
llas frente a nuestras costas. Esta declaración fue inicialmente juzgada como ilusoria y desmedida por muchas potencias marítimas; sin embargo, tras larga y ardua lucha vino a cristalizar en 1982 en la Convención de las Naciones Unidas sobre el Derecho del Mar, la cual entró en vigencia en 1994. Éste es un logro histórico y de nivel mundial; y es nuestro. Con él establecimos una tendencia que ya nadie discute: que los países ribereños tienen derechos sobre sus aguas aledañas y fondos marinos; y que los recursos del mar son finitos y deben ser conservados, protegidos y administrados racionalmente.

También quisiera destacar la creación de la Comisión Permanente del Pacífico Sur, nacida al alero del mismo acuerdo anterior, al que luego se incorporara Colombia. Esta institución constituye en sí misma una instancia de cooperación en el ámbito marítimo regional, con un tremendo potencial para consolidar la presencia de los países ribereños y su proyección hacia la cuenca del Pacífico. Este organismo, y su actualización si fuera necesaria, ofrece una plataforma en torno a la cual podríamos intensificar una acción conjunta en beneficio de intereses marítimos, tales como la conservación de los recursos vivos, la protección del medio ambiente marino, la investigación científica del fenómeno del Niño, que tanto nos está afectando, etc.

En esta misma línea, debemos mencionar el Acuerdo de Galápagos, firmado en agosto del año 2000, dentro de la estructura de la Comisión Permanente del Pacífico Sur, para la protección y administración de pesquerías en el Pacífico. Este acuerdo marco, nos abre un amplio campo de cooperación para desarrollar actividades de importancia económica y científica para ambos países.

Por otra parte, estimo que existe un tema de gran actualidad que podría convocarnos a una mayor cooperación entre nuestras Armadas. Me refiero a lo que se ha dado en llamar las "nuevas amenazas" y las responsabilidades que ellas nos imponen.

Es claro que el terrorismo, el narcotráfico, el tráfico de armas, la delincuencia organizada y otros crímenes transnacionales atacan simultánea e integralmente a las personas, a los Estados y al sistema internacional en su conjunto. Estas organizaciones, con alarmante frecuencia, pueden alcanzar capacidades militares operativas que desafían a las de las policías e incluso a las de las propias Fuerzas Armadas. O bien, amparándose en la sorpresa y 
Visión estratégica

de la Armada

de Chile en las facilidades que ofrece la propia democracia, pueden asestar un golpe asimétrico en fuerza, pero letal en sus efectos, como ocurrió el 11 de Septiembre, en Estados Unidos.

Esta nueva situación tiende a difuminar los límites que establecían nuestros espacios de acción institucional, haciendo más difícil diferenciar la Seguridad de la Defensa. Creo que es un tema al que debemos dedicar tiempo y esfuerzo intelectual, para establecer nuevas formas de cooperación e interacción, que nos permitan enfrentar con armas renovadas y eficaces estos desafíos, de los cuales ningún país puede considerarse a salvo ni menos ignorar.

\section{LA RELACIÓN ENTRE NUESTRAS ARMADAS}

En lo que respecta específicamente a nuestras Marinas, tenemos un interesante rol que jugar en el Pacífico Sur y lo haremos mejor si actuamos en conjunto. Actualmente, así lo estamos haciendo, y en una forma que hace algunos años habría sido impensable. A las reuniones de Altos Mandos entre Fuerzas Armadas que veníamos sosteniendo desde hace años, hemos agregado nuevas actividades de gran significación entre ambas Marinas, como es la $1^{a}$ Reunión de Estados Mayores sostenida en el mes de abril recién pasado, y cuyos frutos podremos valorizar a corto plazo.

Asimismo, estamos adquiriendo una creciente fluidez en el ámbito de las reuniones técnicas para tratar asuntos específicos, como son las Conferencias Bilaterales de Inteligencia, las reuniones entre Autoridades Marítimas y entre Comandantes en Jefes de Zonas Navales fronterizas. A esto se agregan las actividades oceanográficas que estamos realizando en conjunto ambas Marinas, como son los cruceros combinados de investigación del fenómeno del niño y los ejercicios de alarmas anti Tsunamis. Por su parte, los ejercicios binacionales y las visitas de unidades navales también muestran un aumento significativo.

En lo personal, lo que más valoro es el creciente intercambio de oficiales y cadetes navales. Los jóvenes oficiales chilenos que se han embarcado en vuestras unidades fluviales, y los oficiales peruanos que han navegado en nuestros buques en la zona de Puer- 
to Montt, sumado al intercambio de cadetes en competencias deportivas y oficiales alumnos en las respectivas Escuelas de Guerra, representan la promesa de un mejor conocimiento y mayor aprecio entre nosotros.

Estamos optimistas y determinados a seguir contribuyendo en este esfuerzo mutuamente conveniente. Tenemos la convicción de que el mundo que se está conformando, lo está haciendo sobre la base de la acción concertada de grupos de países unidos por la cultura, la religión, la proximidad física y por intereses nacionales específicos.

No nos corresponde a nosotros, como Fuerzas Armadas, decidir los énfasis ni la intensidad del proceso de integración y cooperación entre nuestros Estados, puesto que aquello es una decisión esencialmente política. No obstante, podemos contribuir con nuestro grano de arena a este noble objetivo que, a nuestro juicio, cada vez se hace más demandante. Si queremos ser actores y no meros espectadores del arrollador y despiadado proceso de globalización que estamos viviendo, debemos unirnos, porque la unión hace la fuerza.

\section{REFLEXIONES FINALES}

Para finalizar, quisiera invitarlos a reflexionar sobre algunas de las ideas que he enunciado en esta grata visita a la Escuela Superior de Guerra Naval del Perú.

En primer término, sostengo que nuestra historia común, con sus encuentros y desencuentros, es el fundamento realista a partir del cual debemos plantearnos un futuro con otra perspectiva, asumiendo ese pasado y brindándonos la libertad de elegir hacia dónde queremos ir, sin ataduras ni recelos. Esa elección de futuro debe ser complementada con el mayor conocimiento mutuo que podremos desarrollar en el tiempo, convencidos que de esta forma estaremos contribuyendo más y mejor al logro de nuestros respectivos proyectos nacionales.

Por su parte, en el caso de Chile, su Proyecto Nacional está íntimamente asociado con el entorno internacional. Entendemos que lo que sea bueno para nuestros vecinos, para la región y el hemisferio, redundará en un beneficio para nosotros. Por eso, es- 
Visión estratégica

de la Armada

de Chile tamos convencidos de que el éxito de nuestro proyecto se lograría de mejor forma con una mayor cooperación e integración con el Perú. Creemos que más allá de contar con determinadas riquezas, ese éxito pasa por optimizar nuestra capacidad de gestión, y por una mirada de futuro que nos permita hacer converger nuestros proyectos nacionales hacia objetivos comunes.

En cuanto a la Armada de Chile, estamos conscientes del papel que debemos jugar en la consecución de los objetivos de nuestro Proyecto Nacional. Somos uno de los instrumentos de la Estrategia que cumple funciones más allá de la defensa del territorio, apoyando la Presencia Internacional de Chile, y protegiendo aquellos intereses que se vean afectados por las amenazas a la paz, tarea que por cierto debe ser efectuada en conjunto con otros Estados. Estas tareas, se enmarcan en uno de los tres vectores de nuestra Estrategia Naval, aquel que busca la participación y promoción de un interés que es común para los Estados Marítimos, cual es preservar la seguridad, la estabilidad y el orden internacional en el mar; es decir, mantener la paz.

Alrededor de estos intereses, que estimamos comunes, ambos países podemos ir paulatinamente incrementando nuestras áreas de cooperación en el ámbito marítimo y naval, orientados al Océano Pacífico. Fuimos visionarios en 1952, al establecer el límite de 200 millas náuticas de jurisdicción marítima. Ahora, podríamos explorar nuevas áreas de cooperación marítima, y ser prospectivos respecto de las nuevas amenazas que se incorporan en los albores de este siglo. Amenazas cuya neutralización tiende a difuminar las fronteras entre Seguridad y Defensa, presentando a las Fuerzas Armadas nuevas y desafiantes responsabilidades.

Para asumir estas nuevas responsabilidades, tenemos una tarea que está en ciernes, pero de la cual hemos dado muestras de una adecuada voluntad para acometerla: me refiero al incremento de la cooperación y entendimiento mutuo.

Espero y confío en que la creciente relación entre nuestras Armadas continúe siendo uno de los pilares en el que se sustentará la sólida amistad entre Chile y Perú. En nuestras manos está que esta amistad fructifique y converja hacia un futuro esplendoroso para nuestras marinas y nuestros países. 